\title{
Diabetes-related health beliefs explored in low-income Latinos
}

\author{
Marciel A. Klenk \\ Anna C. Martin \\ Anna Olivares \\ Amy B. Joy \\ Hugo Quiñonez-Melgar \\ $\nabla$
}

We conducted focus group dis-cussions and a survey to explore diabetes-related health beliefs and to assess educational needs among low-income Latino adults, primarily of Mexican descent. We found that many low-income Latinos served through UC Cooperative Extension programs may be at high risk of developing diabetes. At the same time, many of those at risk lack awareness of risk factors for dia-betes, have never been screened and do not know where to go for advice. Although some were unsure of the true causal connection, $64 \%$ of the focus group participants had heard that susto (stress or strong emotion) might be related to onset of the illness. In addition to increas-ing awareness of the disease and reducing barriers to care, nutrition educators can help program partici-pants by identifying ways to alleviate the effects of stress and lower the risk of diabetes through a healthy lifestyle.

B etween 1990 and 1998, California experienced an increase of $67 \%$ in the prevalence of diabetes, from $4.9 \%$ to $6.9 \%$ of the total population (Mokdad et al. 2000). Experts believe this trend, also observed nationwide, is due to the obesity epidemic, as excess body weight is a major risk factor for type 2 diabetes. Since diabetes can damage almost every major organ and shorten the life span by an average of 10 to 15 years, the economic implications of this trend are profound. Currently the estimated cost

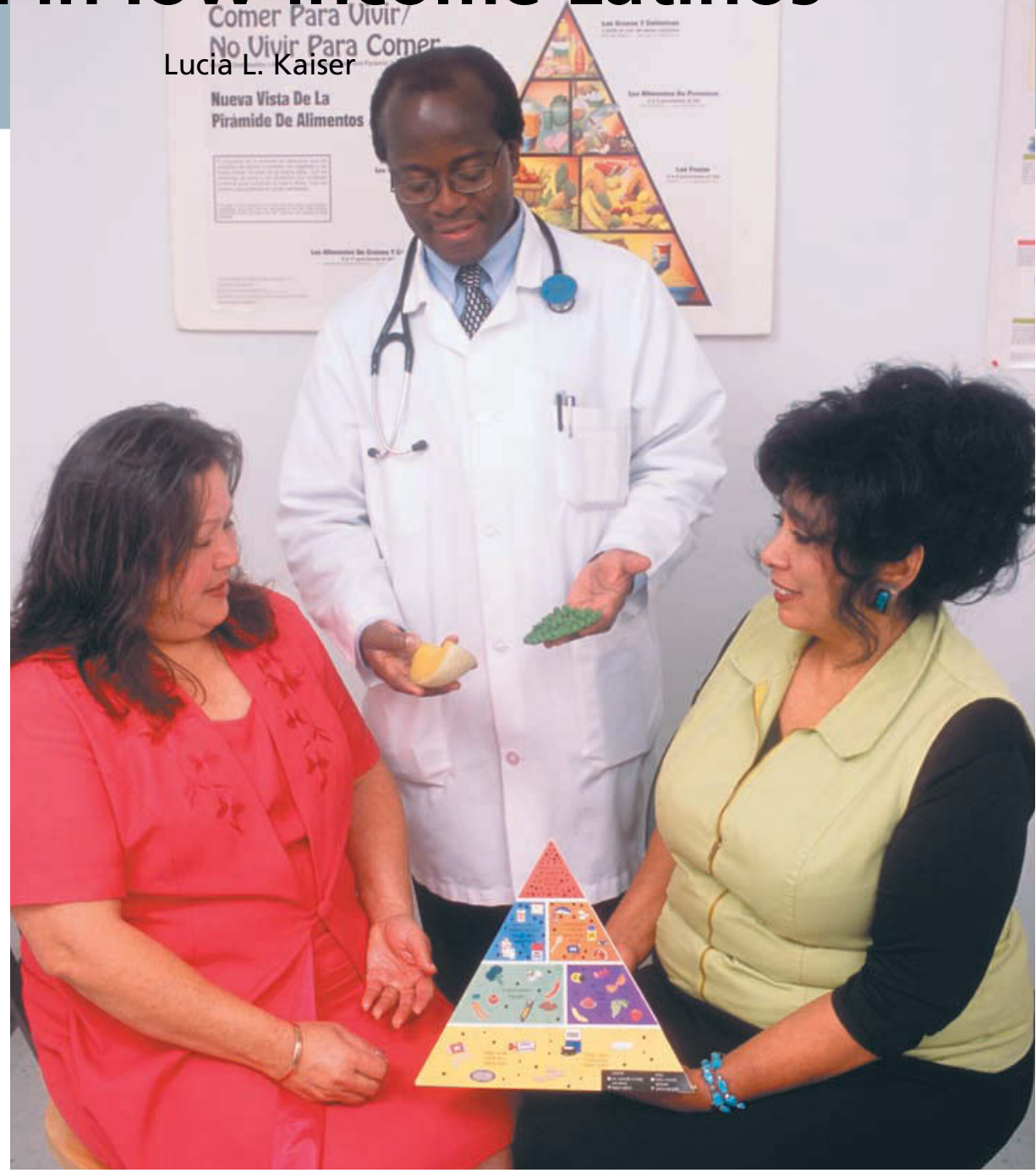

At the Fifth Street Medical Center in Stockton, UC Cooperative Extension nutrition educator Anna Olivares (right), reviews healthy food choices with Blanca Reyes (left) and Dr. Kwabena Adubofour. Reyes, a native of Siñaloa, Mexico, was diagnosed with gestational diabetes during her last pregnancy and now suffers from type 2 diabetes.

of diabetes to the nation is over $\$ 105$ billion annually (Diabetes Research Working Group 1999). More than 1 out of 10 U.S. health dollars in 1999 were spent on diabetes.

Diabetes refers to a class of diseases, characterized by high blood glucose levels. All types of diabetes are probably influenced by genetic, environmental and/or lifestyle factors (Diabetes Research Working Group 1999). Between $5 \%$ and $10 \%$ of all cases are classified as type 1 diabetes, which usually develops during childhood or adolescence. The onset of type 1 diabetes appears to be linked to an autoimmune process that destroys the insulin-producing beta cells in the pancreas. People with type 1 diabetes must take insulin by injections or pump in order to avoid unconsciousness, coma and death.

Between $90 \%$ and $95 \%$ of all diabetes cases are type 2 , which generally develops later in life. Type 2 diabetes is characterized by increased resistance to insulin in the body and/or a relative deficiency of insulin. Obesity and lack of physical activity increase the chances of developing type 2 diabetes in genetically susceptible people.

A third form of the disease is gesta- 


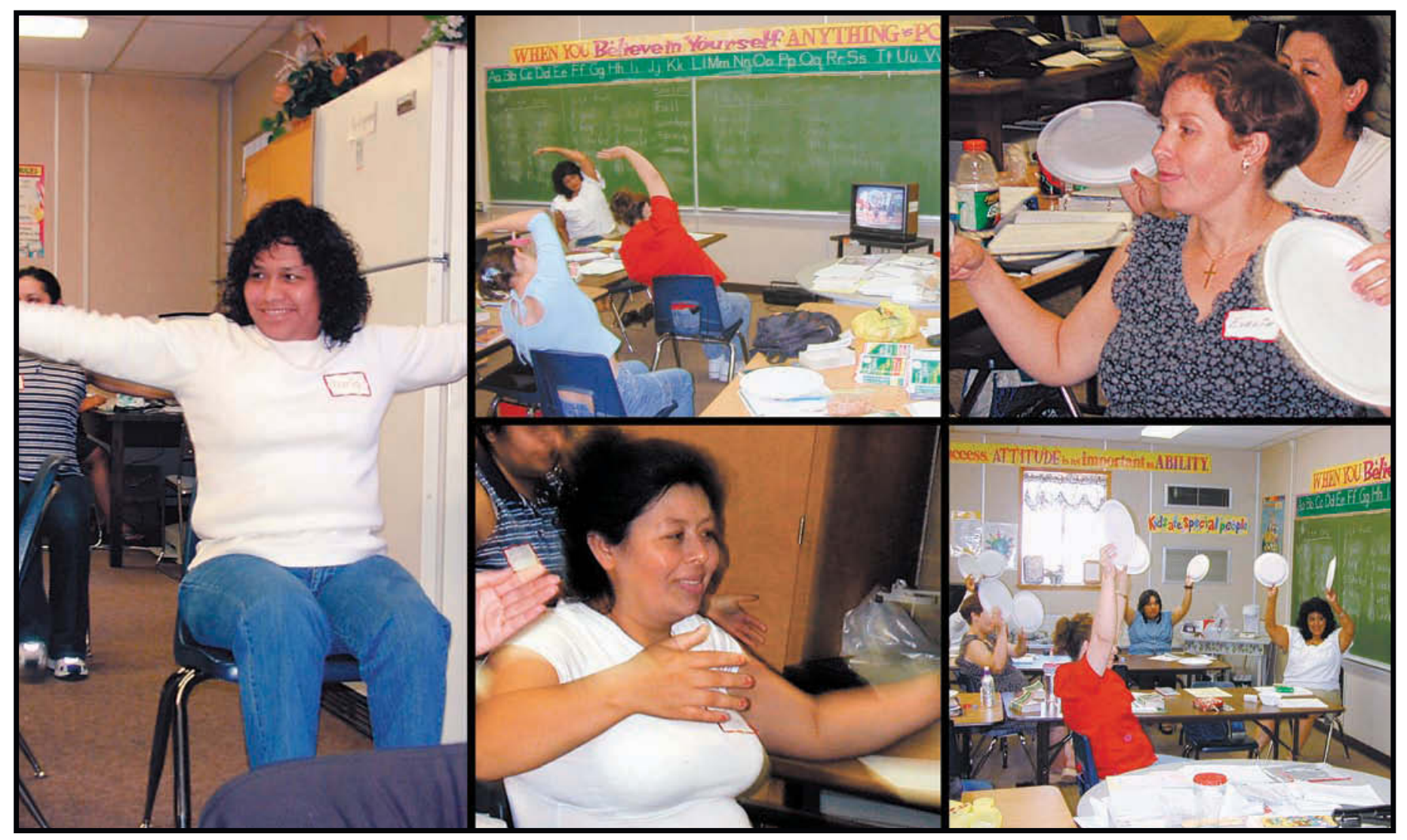

To promote healthier lifestyles among Latinos at-risk of diabetes, UC Cooperative Extension advisors developed the "Take Care of Yourself: Diabetes Awareness and Education" curriculum. In Escalon, Anna Olivares conducted a diabetes-prevention workshop for Spanish-speaking women at the Dent Elementary School's Family Literacy Program. Participants enjoyed "chair dancing" to music from around the world.

tional diabetes, insulin resistance that some women experience during pregnancy. Although gestational diabetes usually disappears after delivery, half of women who have had this form of diabetes will develop type 2 diabetes later in life. Furthermore, impaired glucose tolerance (IGT) is a condition where blood glucose levels are higher than normal but not high enough to be classified as diabetes. Up to half of the people with IGT may develop type 2 diabetes in 2 to 12 years (Bourn 1996). Diabetes is a progressive, chronic disease that cannot be cured. However, careful management through diet, physical activity and usually medication can decrease the risk of complications and improve the quality of life.

\section{Prevalence and prevention}

Recently, a multicenter, randomized, controlled trial showed that intensive lifestyle changes can reduce the incidence of type 2 diabetes over a 3-year period by as much as 58\% in an ethnically diverse population at high risk (Diabetes Prevention Program Research Group 2002). These lifestyle changes include 30 minutes of moderate physical activity on most days of the week, a weight loss of $7 \%$ and a low-fat diet. Sustaining intensive lifestyle changes over the long term may be difficult, but even if the onset of diabetes could be delayed by only a few years, the personal benefits and health care cost savings would be substantial.

In the United States, the prevalence of type 2 diabetes is significantly higher in Latinos, American Indians and African Americans than in non-Latino whites. Mexican Americans are 2.5 times more likely to develop diabetes than non-Latino whites (Haffner 1998). Mexican Americans also tend to have higher fasting blood-glucose levels and suffer more diabetes-related complications than non-Latino whites (Harris et al. 1999; Hosey et al. 1998).

These disparities could be due to a more severe form of diabetes, barriers to health care or both. Culturally sensitive interventions have been successful in improving diabetes knowledge, dietary management and blood sugar control in Latinos with diabetes (Brown and Hanis 1995; Vazquez et al. 1998).
To develop effective programs that increase awareness and encourage early screening, more research is needed on health beliefs and barriers to care in minority populations. The purpose of this study was to explore the perceptions, beliefs, attitudes and behaviors related to diabetes and assess the need for education in low-income Latino adults, primarily of Mexican descent.

In the first stage of our study, four focus group discussions were conducted among Latino adults with type 2 diabetes who resided in three California counties: Napa (two groups), San Joaquin (one group) and Stanislaus (one group). This provided a mix of rural and urban populations in Central and Northern California. In the second stage, a short questionnaire, based on themes discussed during the focus groups, was administered to Latino participants in the Expanded Food and Nutrition Education Program (EFNEP) in San Joaquin and Stanislaus counties. Most of the EFNEP participants were not expected to have diabetes, since the program is designed to deliver 


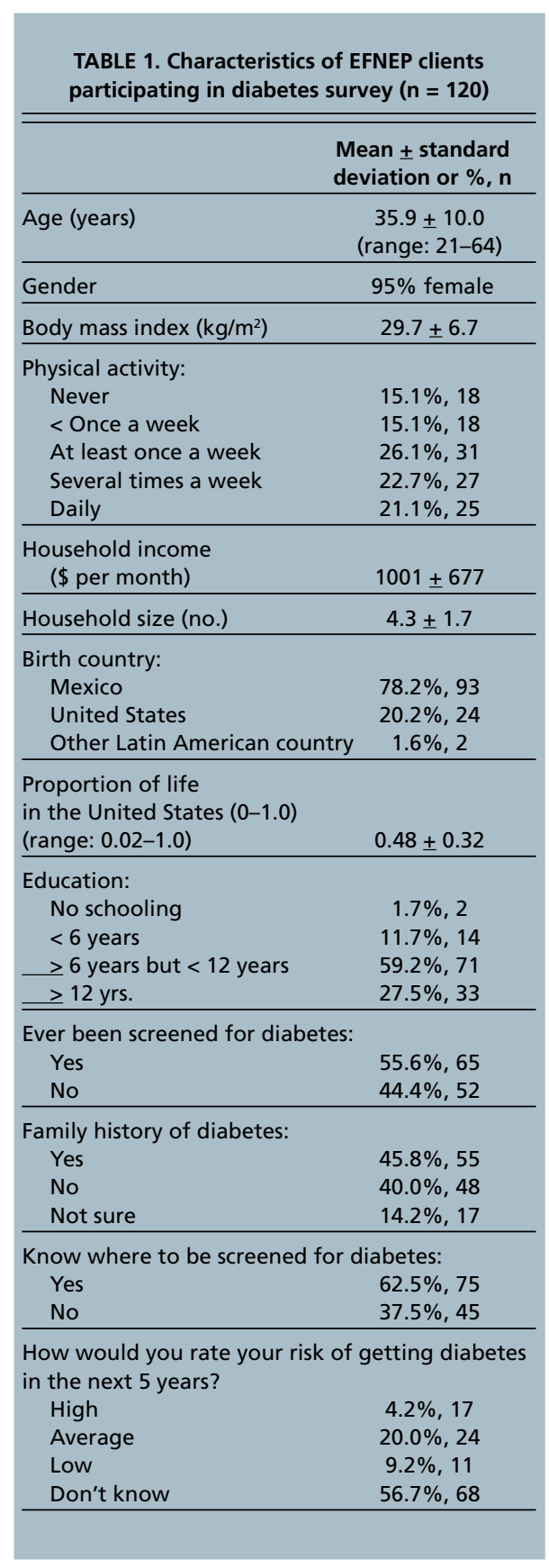

basic nutrition education to the general population. The UC Davis Human Subjects Review Committee exempted the protocol from full review.

\section{Focus group discussions}

UC Cooperative Extension (UCCE) staff recruited the participants for the focus groups through agency staff from local health clinics in the three counties. All participants were adults (ranging in age from about 30 to 75 years), selfidentified as Mexican American, Latino or Hispanic, and had been diagnosed with diabetes. Subjects were offered refreshments and a $\$ 15$ gift certificate as an incentive to participate in the discussion.

The questioning guide and protocol were developed based on Krueger's (1994) guidelines. The questions explored the perceived causes of diabetes, context surrounding the diagnosis, the participant's current experience with diabetes, intervening conditions, actions taken as a result of diabetes and consequences of those actions. The first author, who is bilingual and experienced in leading focus groups, moderated all four groups in Spanish. The co-moderators, who handled recruitment and took notes, were bilingual Latinas. Each session lasted about 90 minutes and was audiotaped, translated and transcribed by the comoderators. A second native speaker (H. Melgar) checked the tapes and transcripts. Two researchers (L. Kaiser and $\mathrm{H}$. Melgar) independently coded, sorted and categorized segments of the transcripts using an approach termed axial coding (Strauss and Corbin 1990).
Interobserver ratings agreed in more than $82 \%$ of the cases. The remaining cases were re-examined and discussed until consensus was reached.

Participants. Twenty-eight Latinos (23 females, 5 males) participated. Most were born in Mexico (only two were U.S. born). The participants had diabetes for an average of 6.5 years (from 2 months to 26 years). Most were currently married (22 married, three widowed, two single, one divorced). Fifty-seven percent $(n=16)$ reported that at least one close family member (parent, grandparent, sibling or child) also had diabetes.

Themes. A major theme was the belief that shock, worry, anger, sadness or an emotional event (termed here as susto) causes diabetes. Although some were uncertain about a causal relationship, most (64\%) had heard that susto might be related to the onset of the illness. The specific reasons why people thought diabetes might result from susto were not discussed. However, people who believed susto might be a factor cited both immediate cause-and-effect examples such as death of a parent or an accident, as well as more prolonged periods of sadness and depression related to coming to the United States. Other factors mentioned less often than susto included obesity (21\%), fatty food $(21 \%)$, sugar or soda $(21 \%)$, an unbalanced diet (11\%) and medications (3\%). While more than half had at least one family member with diabetes, only three subjects $(11 \%)$ mentioned family traits or genetics as causal. In each of these cases, several close family members had diabetes.

\section{Focus group responses: Latinos and diabetes (Napa and Stanislaus county women)}

"My mother told me I shouldn't take insulin, it is bad, dilutes the blood."
"Some people are afraid of other people knowing that they have diabetes. I was one of those people but my doctor explained it to me and I understood that it is a disease that we can control."
"I think that I got it (diabetes) because of anger (coraje). Nobody in my family has it. I think it's coraje and fright (susto) together that do it."
"We Hispanics eat a lot of fat, and we eat very few vegetables. We eat more fat - like pozole (a stew with chili, corn and pork) - pure fat, pork skin and from there the diabetes is due to the fat."
"Have less worries. Have a good outlook. Resign ourselves to the fact that God knows what he is doing. Get medical care and abide by the advice that the doctor gives you." 
Other health beliefs and attitudes about diabetes were mentioned. Some subjects had heard from family members that insulin dilutes the blood or causes blindness. One woman mentioned that she had been ashamed to let others know she had diabetes.

Several people voiced the belief that everyone - especially Mexicans - has diabetes, but in some people the disease just develops faster. Religious beliefs were mentioned but with a realism about internal control over the disease. In other words, while becoming ill may be perceived as part of God's design, the individual should not abandon self-care.

\section{EFNEP survey}

Based on the focus group themes, a set of questions was developed, translated into Spanish, cross-checked by a diabetes educator in Napa County, and tested in a discussion group with the target audience. Starting in early 1999, the UCCE nutrition educators in San Joaquin and Stanislaus counties contacted schools and family community centers to schedule their usual EFNEP classes. The first eight classes that targeted Latino adults were selected for the survey. (Classes scheduled at drug rehabilitation programs were not included in the sample.) Upon enrollment in EFNEP, 132 participants completed the standard EFNEP family record form, food behavior checklist and our supplemental set of 40 diabetes-related questions. The survey was self-administered and mainly included agree/disagree/ not sure response categories.

To get a measure of acculturation, data was collected on country of birth and years of residence in the United States. A portable electronic scale and stadiometer were used to weigh and measure the participants. Nine percent of the participants $(n=12)$, who reported having diabetes, were excluded from the data analysis. Descriptive statistics were prepared using SAS, Version 8.0 (SAS Institute, Cary, N.C., 1999-2000). The relationship between health beliefs and acculturation was examined using Spearman's correlation, with a $P$ value of less than 0.05 considered significant.

Participants. Although the overall profile (ethnicity, overweight, positive family history, older age, sedentary lifestyle) suggests that many participants in EFNEP classes may have significant risk factors for type 2 diabetes (table 1), 44\% reported never having been screened. Thirty-seven percent did not know where to be screened for diabetes, and only $14 \%$ considered themselves to be at high risk.

Health beliefs. Among this population of Latinos, emotional stressors were widely believed to play a role in the onset of diabetes ( $44 \%$ agreed)

(table 2). Many were uncertain $(71 \%)$ or agreed $(17 \%)$ that insulin injections might be harmful. Only 38\% believed that diabetes cannot be cured. However, many did not feel that diabetes is inevitable (48\%) and claimed they would not be afraid to be screened $(60 \%)$. The proportion of one's life spent in the United States was positively correlated with many of the items, suggesting that more acculturated Latinos have greater knowledge about diabetes.

\section{Nutrition education implications}

Our results are consistent with other studies conducted elsewhere among Latinos. In South Texas, Mexican Americans with type 2 diabetes commonly cited provoking events, in addition to biomedical factors, as a cause of their illness (Hunt et al. 1998). The belief that fright, anger and other strong emotions might cause diabetes appears to be more widely held among Mexicans and Guatemalans than among Puerto Ricans and more-acculturated Latino subgroups (Weller et al. 1999). Traditional folk beliefs relate susto (stress or strong emotion) to the onset of depression and malaise in general, not just diabetes (Kittler and Sucher 1995). The association of imbalance (emotional or physical) with illness and disease may actually have its origins in the ancient pre-Columbian medical-food system (Grivetti 1992). Although stress is not believed to be causally related to diabetes, it may act as a trigger in people who have undiagnosed diabetes and result in a worsening of the condition so that the illness becomes apparent.

In Connecticut, greater diabetes knowledge was also positively correlated with longer residence in the United States. In Illinois, health providers have

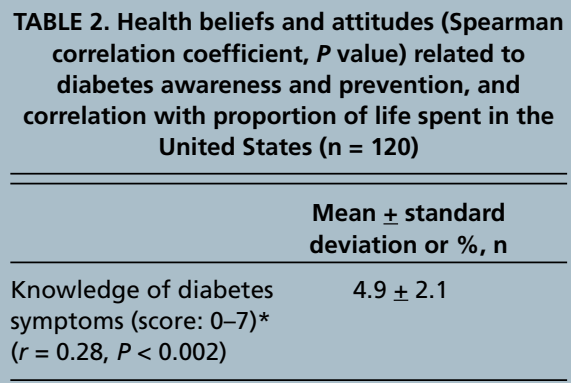

Taking insulin injections can cause blindness

or thin the bloodt $(r=0.29, P<0.002)$

$\begin{array}{ll}\text { Agree } & 17.5 \%, 21 \\ \text { Not sure } & 71.7 \%, 86 \\ \text { Disagree } & 10.8 \%, 13\end{array}$

Shock, anger or worries are the most important causes of diabetes $(r=0.18, P<0.05)$ Agree $\quad 44.2 \%, 53$ Not sure $\quad 36.7 \%, 44$ Disagree $\quad 19.2 \%, 23$

I am afraid to get screened for diabetes, because I wouldn't want others to know I have this disease (NS $\ddagger)$

$\begin{array}{ll}\text { Agree } & 19.2 \%, 23 \\ \text { Not sure } & 20.0 \%, 24 \\ \text { Disagree } & 60.8 \%, 73\end{array}$

There is little one can do to avoid getting diabetes $(r=0.33, P<0.0002)$ Agree $15.8 \%, 19$ Not sure $\quad 36.7 \%, 44$ Disagree $\quad 47.5 \%, 57$

There is a cure for diabetes $(r=0.19, P<0.04)$ Agree $27.5 \%, 33$ Not sure $\quad 34.2 \%, 41$ Disagree $\quad 38.3 \%, 46$

Having diabetes would mean that you can no longer work or carry heavy things $(r=0.29$, $P<0.001)$

$\begin{array}{lc}\text { Agree } & 4.2 \%, 5 \\ \text { Not sure } & 36.7 \%, 44 \\ \text { Disagree } & 59.2 \%, 72\end{array}$

Following a diabetic diet would mean that you cannot eat the foods you like $(r=0.18$, $P<0.05)$

$\begin{array}{ll}\text { Agree } & 40.8 \%, 49 \\ \text { Not sure } & 23.3 \%, 28 \\ \text { Disagree } & 35.8 \%, 43\end{array}$

Getting regular exercise can lower my risk of getting diabetes (NS)

$\begin{array}{ll}\text { Agree } & 61.3 \%, 73 \\ \text { Not sure } & 26.1 \%, 31 \\ \text { Disagree } & 12.6 \%, 15\end{array}$

Eating more fiber can lower my risk of getting diabetes (NS)

$\begin{array}{lr}\text { Agree } & 65.6 \%, 78 \\ \text { Not sure } & 26.1 \%, 31 \\ \text { Disagree } & 8.4 \%, 10\end{array}$

* Score based on number of correctly identified diabetes symptoms (thirst, weight changes, headache, dizziness, loss of vision, frequent urination, feeling tired).

† For all knowledge questions (except knowledge of diabetes symptoms), response categories were coded: 1 = agree, 2 = not sure, 3 = disagree.

‡ Not significant. 
similarly encountered negative beliefs among Latinos about insulin (Lipton et al. 1998). These providers have expressed the concern that fear of insulin may pose an even greater barrier to care than lack of financial resources.

Since $95 \%$ of the participants in our survey were female, more research about health beliefs in Latino males is needed. However, since Latino women tend to participate more often in community nutrition education classes, this research has important implications for nutrition educators, especially those working in Cooperative Extension programs. Many low-income Latinos served through Cooperative Extension may have significant risk factors for diabetes, yet lack awareness of risks, have never been screened and do not know where to go for advice. Nine percent of the subjects enrolled in the EFNEP classes that we surveyed reported actually having diabetes. Preliminary unpublished results from surveys conducted in New York, Pennsylvania, Arkansas, Maryland and Washington also report a diabetes prevalence of $9 \%$ for their EFNEP participants $(n=519)$ and $18 \%$ for participants in the Food Stamp Nutrition Education Program ( $\mathrm{n}=321$ )(unpublished data, Butkis 2002). While diabetes education is a nontraditional area for extension educators, a recent e-mail survey of food and nutrition extension contacts in 38 states found that $90 \%$ are already involved in some level of diabetes programming (unpublished data, Archuleta 2002).

The Health Beliefs Model is a theoretical framework that describes how to motivate people to take a specific action or change a behavior to improve their health (Rosenstock 1990). Using the Health Beliefs Model and findings from this study, EFNEP educators can develop an effective, culturally relevant approach for Latinos to:

- Increase awareness of risk factors (perceived risk).

- Inform people about the consequences of diabetes (perceived severity).

- Reduce barriers to seeking care and adopting a healthy lifestyle (perceived barriers).
- Increase awareness that physical activity, a low-fat diet and weight management may be able to prevent type 2 diabetes (perceived benefits).

Because stress is perceived to be related to health status in this population, culturally sensitive Cooperative Extension programs can help Latino participants by increasing diabetes awareness, reducing barriers to care and identifying ways to alleviate stress and lower risk of diabetes through a healthy lifestyle. "Take Care of Yourself: Diabetes Awareness and Prevention," a new curriculum based on this research, was designed to achieve those objectives in low-income Latino populations, and is now being implemented in San Joaquin and Stanislaus counties and around California.

\section{L.L. Kaiser is Community Nutrition Spe-} cialist, Department of Nutrition, UC Davis; M.A. Klenk is Nutrition, Family and Consumer Science Advisor, UC Cooperative Extension (UCCE) Napa County; A.C. Martin is Nutrition, Family and Consumer Science Advisor, UCCE San Joaquin County;

A. Olivares is EFNEP Nutrition Educator, UCCE San Joaquin County; and A.B. Joy is EFNEP Adult Program Specialist, and H. Quiñonez-Melgar is Postdoctoral Researcher, Department of Nutrition, UC Davis. We gratefully acknowledge the contributions of Nancy Feldman in conducting the research and UC MEXUS in funding the project.

\section{References}

Bourn DM. 1996. The potential for lifestyle change to influence the progression of impaired glucose tolerance to noninsulin-dependent diabetes mellitus.

Diabetic Medicine 13:938-45.

Brown S, Hanis CL. 1995. A communitybased, culturally sensitive education and group-support intervention for Mexican Americans with NIDDM: A pilot study of efficacy. Diabetes Educator 21(3):203-10.

Diabetes Prevention Program Research Group. 2002. Reduction in the incidence of type 2 diabetes with lifestyle intervention or metformin. N Engl J Med 346:393-403.

Diabetes Research Working Group. 1999. Summary of the report and recommendations of the Congressionally established Diabetes Research Working Group. www. diabetes.org/ ada/drwg/drwgsummary.html.

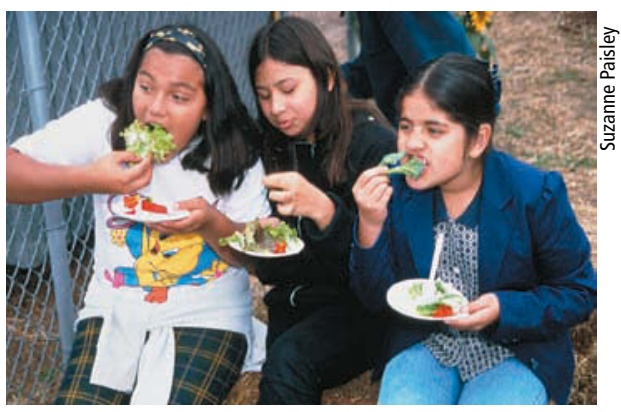

Many low-income Latinos served by UC Cooperative Extension may have significant risk factors for diabetes, yet lack awareness and have not been screened. Early intervention, such as teaching young people healthier eating habits, is critical for preventing and managing type 2 diabetes.

Grivetti LE. 1992. Prescientific origins of nutrition and dietetics. Nutr Today (May/ June):13-25.

Haffner SM. 1998. Epidemiology of type 2 diabetes: Risk factors. Diabetes Care 21 Suppl 3(4):C3-6.

Harris MI, Eastman RC, Cowie CC, et al. 1999. Racial and ethnic differences in glycemic control of adults with type 2 diabetes. Diabetes Care 22(3):403-8.

Hosey GS, Gordon S, Levine L. 1998. Type 2 diabetes in people of color. Nurse Practitioner Forum 9(2):108-14.

Hunt LM, Valenzuela MA, Pugh JA. 1998. Porque me tocó a mi? Mexican American diabetes patients' causal stories and their relationship to treatment behaviors. Soc Sci Med 46(8):959-69.

Kittler PG, Sucher KP. 1995. Latinos. In: Food and Culture in America, 2nd Ed. Belmont, CA: West/Wadsworth. p 253-314.

Krueger RA. 1994. Focus Groups: A Practical Guide for Applied Research. Thousand Oaks, CA: Sage. 255 p.

Lipton RB, Losey LM, Giachello A. 1998. Attitudes and issues in treating Latino patients with type 2 diabetes; views of healthcare providers. Diabetes Educator 24(1):67-71.

Mokdad AH, Ford ES, Bowman BA, et al. 2000. Diabetes trends in the U.S., 1990-1998. Diabetes Care 23:1278-83.

Rosenstock IM. 1990. The Health Beliefs Model: Explaining health behavior through expectancies. In: Glanz K, Lewis FM, Rimer BK (eds.). Health Behavior and Health Education: Theory, Research and Practice. San Francisco: Jossey-Bass. p 39-62.

Strauss AL, Corbin J. 1990. Basics of Qualitative Research: Grounded Theory Procedures and Techniques. Newbury Park, CA: Sage. $270 \mathrm{p}$.

Vazquez IM, Millen B, Bissett L, et al. 1998. Buena alimentacion, buena salud: $A$ preventive nutrition intervention in Caribbean Latinos with type 2 diabetes. Am J Health Promot 13(2):116-9.

Weller SC, Baer R, Pachter LM, et al. 1999. Latino beliefs about diabetes. Diabetes Care 22:722-8. 